\title{
cmaJOPEN
}

\section{Impacts of the Certificates of Added Competence credentialling program: a qualitative case study of enhanced-skill family medicine practice across Canada}

\author{
Lawrence Grierson PhD, Ilana Allice MA, Alison Baker MD, Alexandra Farag MD, Jesse Guscott MD, \\ Michelle Howard PhD, Margo Mountjoy PhD MD, Henry Y.-H. Siu MD, X. Catherine Tong MD, \\ Meredith Vanstone PhD
}

\section{Abstract}

Background: In 2015, the College of Family Physicians of Canada (CFPC) expanded its Certificates of Added Competence (CAC) program to include enhanced-skill certification in Care of Elderly, Family Practice Anesthesia, Palliative Care, and Sports and Exercise Medicine. We aimed to describe the impact of these 4 CACs on the provision of comprehensive care in Canada, while also identifying the factors of influence that foster these impacts.

Methods: Between September 2018 and June 2019, we conducted qualitative case studies of 6 family medicine practices across Canada, sampled to represent geographic, population and practice arrangement diversity. We developed a framework of relevant factors and their relations to CAC-mediated comprehensive care delivery. We took an exploratory approach to the first 4 case studies, guided by theoretical propositions based on a literature review, and the CFPC's 4 principles of family medicine and goals for practice. The emerging theory was confirmed and adapted through the final 2 explanatory case studies. Data were obtained through semistructured qualitative interviews with enhanced-skill and generalist physicians, specialists, trainees and administrators associated with these cases. We performed a descriptive content analysis, within and across cases.

Results: Interviews with 48 participants showed considerable variation in the way CACs are operationalized related to the specific domain of care, the community, relationships among practitioners, motivations of the practitioner and needs of the patient population. The presence of CAC holders in communities expands the scope of available services, reduces the need for patients to travel and encourages continuity of care; however, comprehensive care may be negatively affected when CAC holders develop enhanced-skill practices according to clinical interests rather than community needs. Factors associated with collaborative care models, practice requirements, remuneration structure, community culture and individual aspirations interact to reinforce or undermine the effectiveness of enhanced-skill practices.

Interpretation: Holders of CACs have a positive impact when they work in collaborative models that align with the needs of communities and that support local generalist family physicians. Health care policies should incentivize CAC activities that contribute to planned care delivery at the practice and community levels.

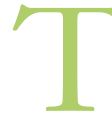
he College of Family Physicians of Canada (CFPC) is the legal certifying body for the practice of family medicine in Canada. It strives for a primary health care system that is "accessible, high-quality, comprehensive and continuous." "Enacting this vision requires family physicians to work collaboratively, leveraging the heterogeneity of individual scopes of practice, so as to extend the comprehensiveness of care in each community. ${ }^{2,3}$ Notably, some family physicians acquire extra skill in a defined domain of care through additional training or practice experience. These people, known as enhanced-skill physicians, provide services that fall outside the typical scope of family medicine (e.g., in-office surgical procedures) or that reflect specialized advances in traditional aspects of primary care (e.g., addictions medicine). By working alongside generalist family physicians, these physicians bring specific expertise where it otherwise might not be available., ${ }^{4,5}$

The Certificate of Added Competence (CAC) is a credential that designates a family physician as having additional

Competing interests: See the end of the article.

This article has been peer reviewed.

Correspondence to: Lawrence Grierson, griersle@mcmaster.ca

CMAJ Open 2021 November 9. DOI:10.9778/cmajo.20200278 
expertise. In 2015, the CFPC built on its Emergency Medicine (EM) certificate, introducing 4 new CACs: Care of the Elderly (COE), Family Practice Anesthesia (FPA), Palliative Care (PC) and Sport and Exercise Medicine (SEM). As of October 2020, 1772 CACs had been awarded in these domains (409 in COE, 600 in PC, 413 in FPA and 350 in SEM).

The CAC is intended to promote enhanced-skill practice that improves the comprehensiveness of primary health care across the country. ${ }^{2}$ Yet, there is speculation that CACs have the unintended effect of encouraging physicians to focus on specific clinical areas in a way that does not contribute to comprehensive generalist care. ${ }^{6-10} \mathrm{We}$ conducted a study of multiple family physician groups aimed at describing the impacts of the newer CACs (COE, FPA, PC

\section{Box 1: Initial theoretical propositions}

The CAC will affect the family physician's skill as a clinician The CAC will affect family medicine's utility as a community-based discipline

The CAC will affect the family physician's utility as a resource to a defined group of patients

The CAC will affect the family physician's relationships with his/ her patients

Interprofessional relationships and arrangements will influence the impacts of CACs within a case

The scope of available services within a community will influence the impacts of CACs within a case

The scope of available services within a community will influence the impacts of CACs within a case

Note: $\mathrm{CAC}=$ Certificate of Added Competence. and SEM) and identifying factors that influence the degree to which family physicians who hold these certificates establish practices that support comprehensive care that is adaptive to the community.

\section{Methods}

\section{Study design}

Between September 2018 and June 2019, in partnership with 4 CFPC leaders, we conducted 6 instrumental case studies, ${ }^{11,12}$ which involved developing a framework of relevant factors and their relations to CAC-mediated comprehensive care delivery.

Without an extant conceptual model, we took an exploratory approach to our first 4 case studies. These were guided by a set of initial theoretical propositions ${ }^{12}$ based on a review of the literature, ${ }^{3-10}$ the CFPC's 4 principles of family medicine, ${ }^{13}$ and the goals for family medicine practice articulated in the CFPC's Patient's Medical Home vision statement ${ }^{1}$ and "Family Medicine Professional Profile" position statement ${ }^{2}$ (Box 1).

As we progressed, our inquiry shifted to a combined exploratory-explanatory focus: the emerging theory of impacts and relevant factors developed through the exploratory cases was confirmed and adapted in different contexts through the final 2 explanatory case studies. ${ }^{12} \mathrm{We}$ treated individual CACs and participant status as a learner as embedded units within each case study, which enabled a more detailed level of inquiry. However, our inquiry coalesced with analyses conducted across case and embedded units so as to identify factors of transcendental relevance (Figure 1).

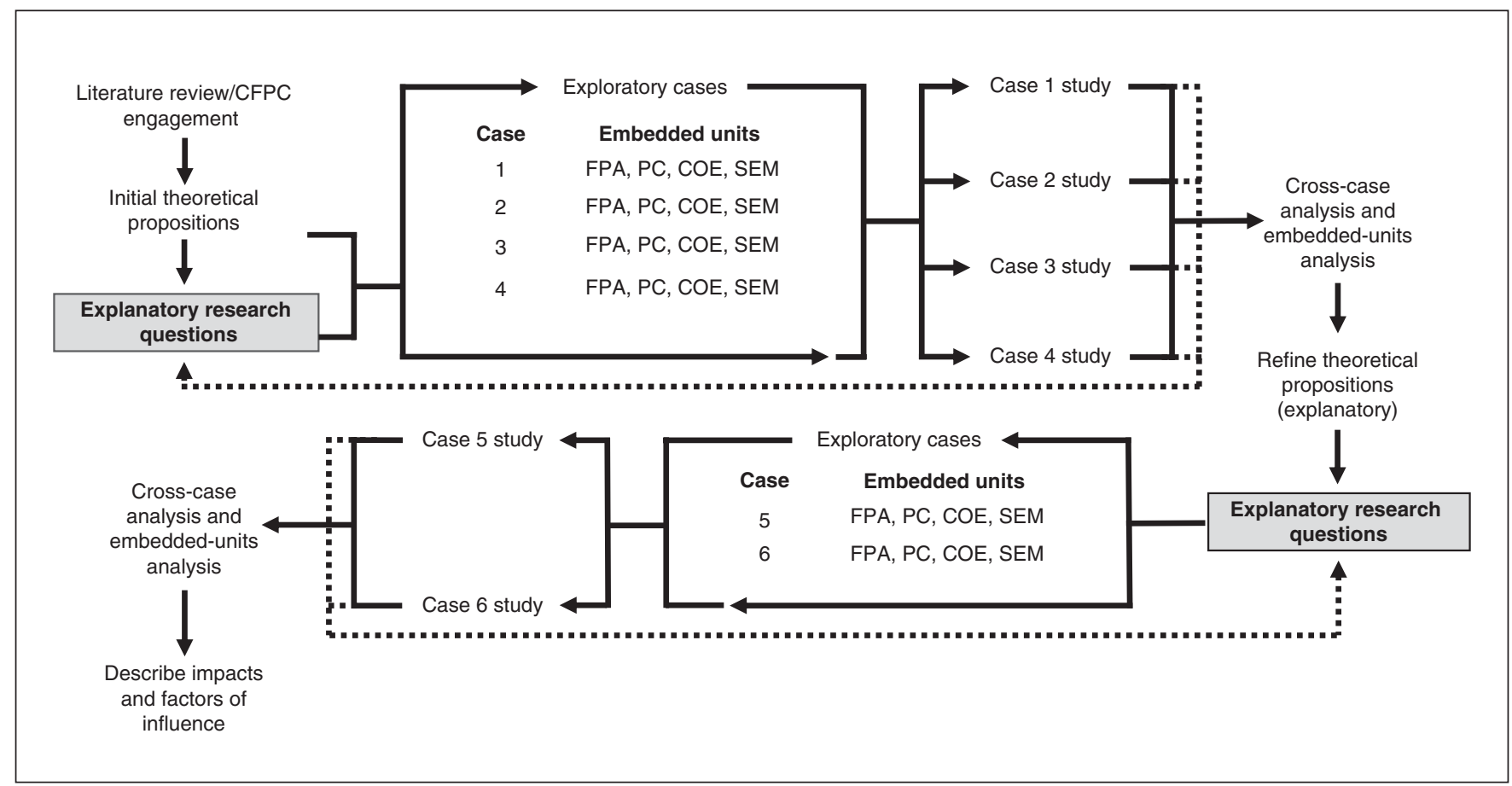

Figure 1: Research design and analytic framework. Note: CFPC = College of Family Physicians of Canada, COE $=$ Care of the Elderly, FPA $=$ Family Practice Anesthesia, PC = Palliative Care, SEM = Sports and Exercise Medicine. 


\section{Case selection}

We defined "case" as a collective of family physicians working with a defined group of patients in an interconnected community. In selecting the exploratory cases, we identified practices in regions characterized by a variety of CAC types and a high density of CACs per population. We sought maximum variation across geography, population density, language, patient population and practice model. We chose the 2 exploratoryexplanatory cases specifically for characteristics that allowed us to test the generalizability of propositions developed through the exploratory cases.

Eligible cases were drawn from any Canadian province or territory. To identify cases, we reviewed practice websites and connected with family physicians knowledgeable about practices in particular regions. We invited these "regional representatives" to discuss the context of family medicine practice in the area and to nominate a collective that exhibited the desired features. These conversations were not entered as data, and not all regional representative conversations yielded theoretically relevant cases.

\section{Participants}

We invited people from each selected case to participate in semistructured interviews. Eligible participants were any professional whose work was related to the case, including CAC holders, other enhanced-skill family physicians, generalist family physicians, resident trainees, specialist physicians and administrative staff. We combined purposeful, criterion and snowball sampling techniques to ensure that we recruited people who could offer perspective on specific aspects of our emerging framework. We ceased recruitment when data sufficiency was determined, related to data completeness within each case. ${ }^{12}$

\section{Data collection}

All interviews were conducted by I.A., a female Masterstrained qualitative researcher employed as a research assistant, between September 2018 and June 2019. Most interviews took place in the participant's workplace, although some were conducted by telephone, according to participant preference. Each participant was interviewed only once, and interviews were designed to last 1 hour.

The interview guide was developed by 3 authors (L.G., I.A. and M.V.) based on key issues identified in the literature, ${ }^{1-10,13}$ by our CFPC partners and by the members of the research team. The guide underwent extensive piloting, starting with the clinician members of the research team and proceeding throughout the first case. The interview guide was refined as data collection progressed and was individualized for each case and type of participant (Appendix 1, available at www.cmajopen.ca/content/9/4/E966/ suppl/DC1). Participants were aware that the interviewer was conducting research funded by the CFPC concerning the CAC program. All interviews were audiorecorded and transcribed verbatim. Field notes were taken throughout the site visit. Transcripts were not returned to participants for comment.

\section{Data analysis}

Once all data were in textual form, analysis began, first withincase and then across cases. We used a descriptive approach to qualitative analysis and a staged process of coding. For the exploratory cases, this involved inductive code generation that simultaneously respected the a priori codes developed from initial propositions or previous cases. Initial coding summarized the content and later condensed it into categories detailing impacts of the CAC program. Subsequent iterations of analysis refined this coding. As our analytic understanding of each case developed, we returned to previous cases to recode for ideas of analytic interest. We used an unconstrained deductive approach to analyze the 2 exploratory-explanatory cases, looking for data that resonated with or refuted the theory. Our goal was theoretical replication across cases. ${ }^{7}$ The coding and analysis were led by L.G., with assistance from I.A. and M.V. Data were managed with NVivo12 (QSR International).

\section{Engagement with College of Family Physicians of Canada}

At regular intervals during the study, we engaged a group of 4 CFPC leaders as research stakeholders to incorporate perspectives from the college's academic, research, education and CAC committees. These people were identified by the college as leading portfolios relevant to the topic of the research. They provided insight that helped us define our initial theoretical propositions, develop the case identification and participant recruitment strategy, and refine our framework at the transition to exploratory-explanatory cases. They facilitated communication with regional representatives and potential practices. This type of engagement is consistent with established approaches to reducing analytic uncertainty, facilitating research logistics and enhancing the likelihood that research findings will be meaningful to knowledge users. ${ }^{14}$

\section{Ethics approval}

This study was approved by the Hamilton Integrated Research Ethics Board (HIREB \#5151). All participants provided informed consent before joining the study.

\section{Results}

Interviews were conducted with 48 participants. No participants withdrew from the study. The practice characteristics of the 6 cases are described in Table 1, and participant characteristics are summarized in Table 2. Interviews lasted about 1 hour.

We identified both positive and negative impacts of the CAC program on the provision of community-adaptive comprehensive care. Across all cases, we documented ways in which CAC holders ground their practice in the needs of the local community and work in collaboration with other health care providers to yield benefit. Benefits included reducing the need for patients to travel for care, serving as a community resource for addressing specific needs, and encouraging continuity of care by supporting the maintenance of the relationship 


\begin{tabular}{|llllll|}
\hline \multicolumn{2}{|l|}{ Table 1: Characteristics of cases } \\
\hline $\begin{array}{l}\text { Case } \\
\text { no. }\end{array}$ & Province/territory & $\begin{array}{c}\text { No. of } \\
\text { physicians }\end{array}$ & Geography & $\begin{array}{c}\text { Institution } \\
\text { type* }\end{array}$ & $\begin{array}{c}\text { Affiliated with } \\
\text { tertiary-level } \\
\text { hospital }\end{array}$ \\
\hline 1 & Ontario & 36 & Urban & Academic & Yes \\
\hline 2 & Manitoba & 51 & Rural & Academic & Yes \\
\hline 3 & New Brunswick & 9 & Urban & Academic & No \\
\hline 4 & Yukon & 20 & Remote & Academic & No \\
\hline 5 & British Columbia & 35 & Rural & Academic & No \\
\hline 6 & Ontario & 100 & Suburban & Community & Yes \\
\hline${ }^{*}$ Academic cases included practices with affiliations to postgraduate training programs. & \\
\hline
\end{tabular}

\begin{tabular}{|c|c|c|c|c|c|c|c|c|c|c|c|c|c|c|}
\hline \multirow[b]{2}{*}{$\begin{array}{l}\text { Case } \\
\text { no. }\end{array}$} & \multirow[b]{2}{*}{$\begin{array}{c}\text { No. of } \\
\text { participants }\end{array}$} & \multicolumn{2}{|c|}{ Gender* } & \multicolumn{6}{|c|}{ CAC domain } & \multicolumn{5}{|c|}{ Type of professional } \\
\hline & & $\mathrm{F}$ & $M$ & $\mathrm{PC}$ & COE & FPA & SEM & EM & AM & $\begin{array}{l}\text { Enhanced-skill } \\
\text { family physician }\end{array}$ & $\begin{array}{l}\text { Generalist } \\
\text { family } \\
\text { physician }\end{array}$ & $\begin{array}{l}\text { Resident } \\
\text { trainee }\end{array}$ & $\begin{array}{l}\text { RCPSC } \\
\text { specialist }\end{array}$ & $\begin{array}{l}\text { Administrative } \\
\text { staff }\end{array}$ \\
\hline 1 & 6 & 4 & 2 & 1 & 1 & 0 & 1 & 0 & 0 & 2 & 0 & 1 & 0 & 0 \\
\hline 2 & 15 & 3 & 12 & 1 & 0 & 3 & 0 & 0 & 1 & 5 & 2 & 1 & 0 & 2 \\
\hline 3 & 8 & 7 & 1 & 1 & 2 & 0 & 0 & 0 & 0 & 0 & 3 & 2 & 0 & 0 \\
\hline 4 & 5 & 1 & 4 & 1 & 0 & 1 & 1 & 0 & 0 & 1 & 1 & 0 & 0 & 0 \\
\hline 5 & 8 & 4 & 4 & 0 & 1 & 0 & 0 & 1 & 0 & 3 & 1 & 1 & 1 & 0 \\
\hline 6 & 6 & 1 & 5 & 1 & 1 & 0 & 1 & 0 & 0 & 1 & 1 & 1 & 0 & 0 \\
\hline Total & 48 & 20 & 28 & 5 & 5 & 4 & 3 & 1 & 1 & 12 & 8 & 6 & 1 & 2 \\
\hline
\end{tabular}

between patients and their primary physician. The CAC program also enhanced the well-being of physicians who were able to establish practices characterized by higher acuity and better remuneration. Similarly, it also reduced the caseload of generalist family physicians who passed patients with more complex medical needs to CAC holders (Table 3, quote 1).

However, when CAC holders do not maintain their own comprehensive family practice, they may not contribute to the provision of comprehensive care in the community. Some CAC holders construct their enhanced-skill practice in a way that reflects their own clinical interests rather than community needs. For example, we heard about SEM holders who have chosen to see only athletes, creating gaps in both generalist care and care for patients who have musculoskeletal issues but are not athletes. In addition, administrative barriers can prevent enhanced-skill physicians from working collaboratively with other family physicians, potentially necessitating multiple transfers of care. This was present in case 5 , in which patients receiving palliative care needed to change care providers multiple times when they moved between the community and the hospital.

\section{Factors of influence}

We identified 3 interactive factors that influence how CAC practice supports or obstructs a community-adaptive form of comprehensive family medicine: the collaborative relationships among practitioners, forces external to the community and the internal community culture.

\section{Collaborative relationships among practitioners}

Our analysis identified 4 organizational models that illustrate the ways in which enhanced-skill family physicians make use of their specialized domains of care: an enhanced scope of services model, a shared-care model, a family-physician-aligned transfer of care model and a specialist-aligned transfer of care model (Table 4).

Some models are strongly associated with particular CACs. For example, many PC holders work in shared-care models, whereas FPA holders often work in a specialistaligned transfer of care model. However, each of the 4 newer certificates provides enough flexibility that its holders may construct an effective practice in any model. For instance, we observed PC holders working alongside oncologists in 


\begin{tabular}{|c|c|}
\hline Quote no. & Quote \\
\hline 1 & $\begin{array}{l}\text { [The availability of PC holders] has kind of taken a rather large load away. ... I get them } \\
\text { [PC holders] involved at some point, because ... I know a fair amount about it [palliative } \\
\text { care], but it rolls off of them much easier than for me. (Case } 4 \text {, participant } 4 \text {, generalist } \\
\text { family physician) }\end{array}$ \\
\hline 2 & $\begin{array}{l}\text { If I see a patient that I think [needs surgery], I will fast-track them to [the orthopedic } \\
\text { surgeon]. Meaning, it doesn't take them } 9 \text { months to see him, it takes them maybe a month } \\
\text { to see him, because I have seen them, I have triaged the patient, and now I know ... it's } \\
\text { time for an assessment in surgery, so they get fast-tracked. (Case } 4 \text {, participant } 3 \text {, SEM) }\end{array}$ \\
\hline 3 & $\begin{array}{l}\text { [CACs] provide another layer of expertise [whereby] they [CAC holders] could handle } \\
\text { something or diagnose something in that area of expertise, and then the patient doesn't } \\
\text { have to go to [the urban centre] or go to a specialist, so the care can happen quicker and } \\
\text { within the same community. (Case } 2 \text {, participant } 9 \text {, generalist family physician) }\end{array}$ \\
\hline 4 & $\begin{array}{l}\text { I couldn't do what I do fee-for-service. For one thing ... geriatricians have actual billing codes } \\
\text { for what we do, family practice does not have billing codes for what I do.... We do } \\
\text { comprehensive geriatric assessments, they take an hour to an hour and a half. ... So, you } \\
\text { couldn't possibly bill family practice codes and do geriatric care. (Case } 3 \text {, participant } 2, \text { COE) }\end{array}$ \\
\hline 5 & $\begin{array}{l}\text { You cannot do shared care and have both doctors paid at the same time in the model that } \\
\text { we're in.... So, if a family doctor wants to do shared care, obviously they're going to bill for } \\
\text { it, that's kind of the point and the incentive, so we kind of work for free in those cases. And, } \\
\text { I do it, to build capacity, but l'm not getting remunerated for it. (Case } 6 \text {, participant } 2, \text { PC) }\end{array}$ \\
\hline 6 & $\begin{array}{l}\text { I tried to start a primary care sports medicine clinic, based out of a physiotherapy clinic, } \\
\text { last fall ... because there has never been a sports doc here before, the community } \\
\text { doesn't have the culture of that, so what I ended up doing was a lot of doubling up on } \\
\text { what the family docs were already doing or on what the [emergency department] was } \\
\text { doing. (Case 4, participant 3, SEM) }\end{array}$ \\
\hline 7 & $\begin{array}{l}\text { I've been able to put "PC" behind my "CCFP," that's it, really.... There's no change in ... I don't } \\
\text { think any of my colleagues even really noticed for the longest time. But they know me by the } \\
\text { fact that I have extra training and I've been able to help them out of difficult situations. That's } \\
\text { how you make the impact. (Case } 2 \text {, participant } 8, P C \text { ) }\end{array}$ \\
\hline 8 & $\begin{array}{l}\text { I enjoy doing the work that I do at the care home. I don't know if I would need or, honestly, } \\
\text { want the extra one [certification, because I think if I did [obtain] the Care of the Elderly } \\
\text { [certification] ... there would probably be a reasonable expectation that I was going to } \\
\text { provide extra services to the region, and I don't know if I have time in my practice or my life } \\
\text { to do that. (Case 2, participant 4, generalist family physician) }\end{array}$ \\
\hline 9 & $\begin{array}{l}\text { It's not to say that I wouldn't value having more people in those [CAC] roles, because if that } \\
\text { improved my access, I would use some of them more. But I trained through a time and } \\
\text { worked in a time [in which] that accessibility wasn't always there. And so, I've learned how } \\
\text { to not need them until I really need them. (Case 3, participant } 7 \text {, generalist family physician) }\end{array}$ \\
\hline
\end{tabular}

specialist-aligned transfer of care models, and an FPA holder treating chronic pain in an enhanced scope of services model.

Distinct benefits were derived from the choice of certain models of care. A family physician who was an SEM holder described how working in a specialist-aligned transfer of care model shortened the wait time for patients referred to see an orthopedic surgeon (Table 3, quote 2).

\section{Forces external to the community}

We identified 4 influential external forces: community need, access to resources, remuneration agreements, and formal privileging and practice requirements.

Community need: For enhanced-skill practices to be effective, they must be operationalized in a way that ensures patient needs related to both generalist primary care and enhanced skills are met. Cases 2 and 4 were located in rural and remote communities that recognized community need for both generalist and enhanced-skill physicians. They formulated community policies to meet these needs, offering only part-time positions in areas of enhanced skill (e.g., FPA) to incentivize clinicians to also maintain comprehensive practices.

Access to resources: The organization and effectiveness of practices that contain enhanced-skills practitioners is influenced by the local availability of resources such as tertiary care services. In rural areas, for example, enhanced-skill providers were actively used as a resource to extend the local scope of available services (Table 3 , quote 3 ).

In urban and suburban communities, access to enhancedskill care was mediated by awareness of and connection to other providers. For example, case 6, located in a suburban context, was a collective of family physicians who practised 


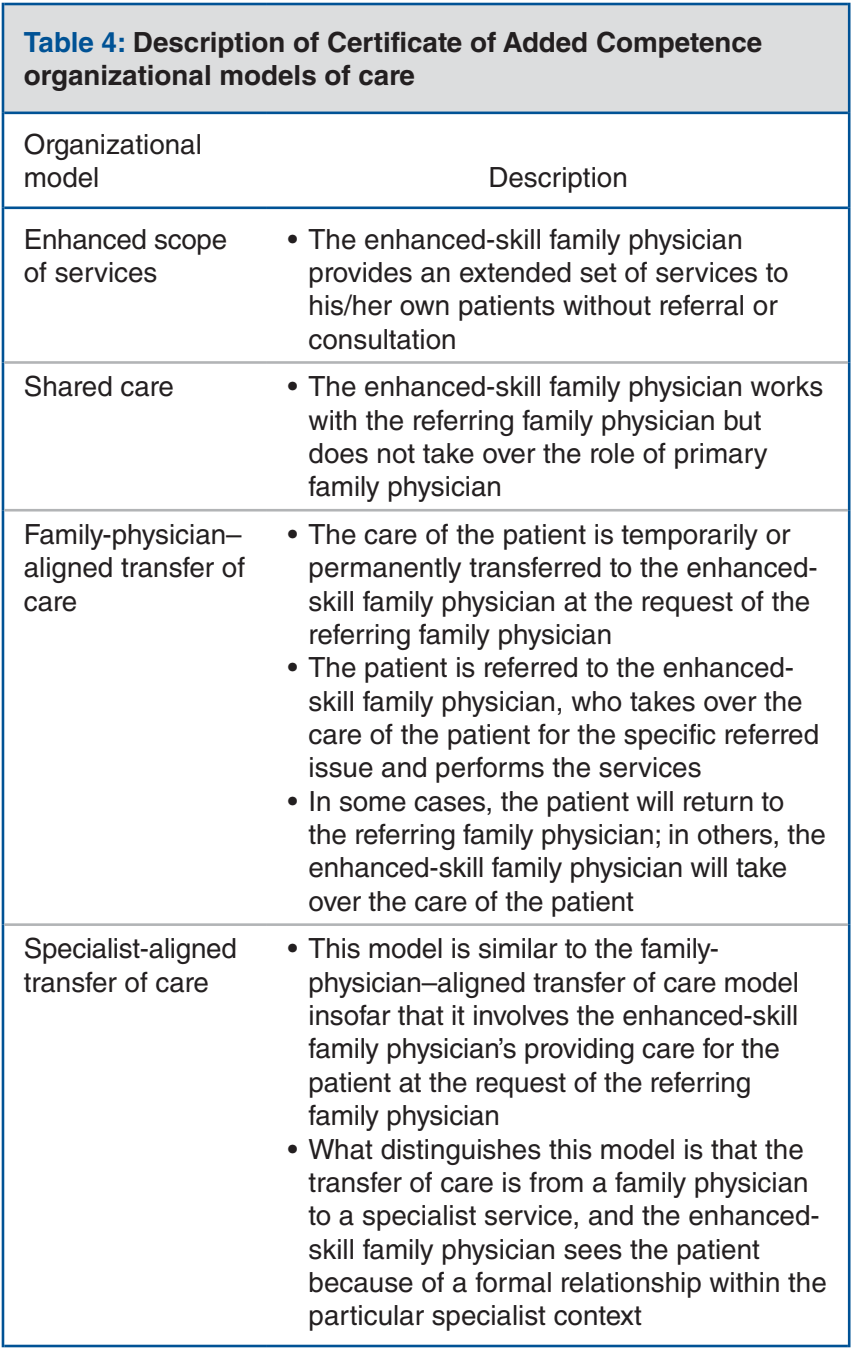

mostly independently but were loosely connected through an optional shared call group. Owing to the high availability of Royal College of Physicians and Surgeons of Canada specialists in this centre and the low connectivity among family physicians, patients were typically referred to specialists rather than enhanced-skill family physicians.

Remuneration arrangement: The arrangement and effectiveness of enhanced-skill practices is influenced by remuneration structures. This is particularly meaningful when financial arrangements facilitate more time with patients. One $\mathrm{COE}$ holder described the necessity of a salaried model to enhanced-skill practice (Table 3, quote 4). The idiosyncrasies of local remuneration models also affect collaboration among physicians (Table 3 , quote 5 ).

Formal privileging and practice requirements: Across the cases, we observed policies that required family physicians to maintain generalist practice or provide inpatient hospital care. For example, in case 4 , family physicians were obligated to participate in a shared inpatient call schedule in order to maintain hospital privileges related to their enhanced-skills work. Choosing not to participate would mean exclusion from financial incentives provided by the regional medical association.
Internal community culture

We identified 2 influential factors related to internal community culture: practice norms, and individual values and attitudes.

Practice norms: In each community we visited, local practice norms had an undeniable influence on the way family physicians were organized and received in the community. Sometimes this created barriers for new CAC holders who wished to establish practices that were atypical for that area (Table 3, quote 6). The expectation or requirement of a credential to substantiate enhanced-skills expertise as opposed to reputational establishment of expertise was another type of practice norm (Table 3, quote 7).

Individual values and attitudes: We noted that individual practitioner values about personal-professional balance and health care delivery served as major contributors to the way in which practice decisions about enhanced-skill work were made (Table 3, quote 8). Some generalist family physicians placed a high value on self-sufficiency and providing timely care (Table 3, quote 9).

\section{Interpretation}

Family physicians across Canada organize their practices, their relationships with other practitioners and their commitments to the communities they serve in a wide variety of ways. This means that the experiences of each CAC holder are unique, and that $\mathrm{CAC}$ practices can have a range of impacts on communities. The impacts and factors of influence presented here reflect our best efforts to capture the elements that have transcendental influence on how family physicians with CACs in the PC, COE, SEM and FPA domains contribute to the delivery of comprehensive, community-adaptive care in Canada., ${ }^{1,2}$ Given the particularities of practice in any one community, family physicians are afforded different levels of opportunity to arrange their practices in ways that are mutually beneficial to both their professional aspirations and comprehensive care within that community. Although the influential factors are delineated above, they should be understood as interactive, covarying as a function of one another.

In any community, there is an opportunity for CAC holders to employ a collaborative model of care that leverages the intersection between the external forces that act on a community and the culture of that community to provide high-quality primary care. When this occurs, the CAC program confers many of the intended benefits. ${ }^{1,2}$ It is an educational policy with the potential to elevate the clinical skill base of a community of physicians, keep patients within their communities, reduce the need for specialist care that may be available only at a distance, improve continuity between patients and physicians, and improve physician well-being. However, the CAC program is not universally beneficial, and it will require additional intervention to meet policy objectives. ${ }^{6-10,15}$ This is particularly true in cases in which CAC holders organize their practice without adequate consideration for community needs. This encompasses instances in which focused practices are established in communities where generalist care is needed or that do not adapt when the health care needs of a community change. 
Strong connections between the CAC holders and other practitioners in each community are essential to making the CAC program work. These connections permit local health care providers to coordinate in order to address patient needs optimally at the community level. The degree to which a CAC holder can organize his or her practice with respect to community need is influenced by local policies that define remuneration models and privileging requirements. Effective shared-care models, for example, are often challenged when the relevant funding model does not account for the way an enhanced-skill family physician may participate in the care of a patient: collaboratively, as a coach or as primary physician. Notably, although higher remuneration and salaried fee structures may be necessary for effective enhanced-skills practice, they may also incentivize focused practice at the expense of generalist care.

\section{Limitations}

There is considerable variation in the way that family physicians across Canada organize their practices, interact with other practitioners and serve their communities. This variation extends to the ways in which CACs are understood and operationalized by individual physicians. As such, we acknowledge that there is no single, all-encompassing way to describe a CAC holder. Rather, these descriptions are intended to offer a broad and transferable perspective. Although our sample size may be seen as a limit to the generalizability of our findings, power in multiple-case study design is enhanced by developing propositions within a case and then testing them across cases. ${ }^{12}$ The number of cases included in this study provides confidence in the findings.

\section{Conclusion}

There are some practice arrangements that facilitate comprehensive care through CAC holders and some practice arrangements that discourage it. In particular, CAC holders tend to have a positive impact on the delivery of comprehensive care when they work in collaborative models that align with the needs of communities and support local generalist family physicians. Indeed, although CAC holders may be most necessary in communities that lack specialist services, they may be most effective in communities with adequate availability of generalist physicians. Health care policies should incentivize CAC activities that contribute to planned care delivery at the practice and community levels.

\section{References}

1. A new vision for Canada: family practice — the patient's medical home 2019. Mississauga (ON): College of Family Physicians of Canada; 2019. Available: https:// patientsmedicalhome.ca/files/uploads/PMH_VISION2019_ENG_WEB_2.pdf (accessed 2020 Oct. 14).

2. Family medicine professional profile. Mississauga $(\mathrm{ON})$ : College of Family Physicians of Canada; 2018. Available: https://www.cfpc.ca/CFPC/media/ Resources/Education/FM-Professional-Profile.pdf (accessed 2020 Oct. 14).

3. Baldwin DC Jr. Some historical notes on interdisciplinary and interprofessional education and practice in health care in the USA. 1996. 7 Interprof Care 2007; 21:23-37.

4. Goldman J, Meuser J, Rogers J, et al. Interprofessional collaboration in family health teams: an Ontario-based study. Can Fam Physician 2010;56:e368-74.

5. Meuser J, Bean T, Goldman J, et al. Family health teams: a new Canadian interprofessional initiative. F Interprof Care 2006;20:436-8.
6. Glazer J. Specialization in family medicine education: abandoning our generalist roots. Fam Pract Manag 2007;14:13-5.

7. Lavergne MR, Goldsmith LJ, Grudniewicz A, et al. Practice patterns among early-career primary care (ECPC) physicians and workforce planning implications: protocol for a mixed methods study. BM7 Open 2019;9:e030477.

8. Coutinho AJ, Cochrane A, Stelter K, et al. Comparison of intended scope of practice for family medicine residents with reported scope of practice among practising family physicians. 7AMA 2015;314:2364-72.

9. Dhillon P. Shifting into third GEAR: current options and controversies in thirdyear postgraduate family medicine programs in Canada. Can Fam Physician 2013;59:e406-12.

10. Shepherd LG, Burden JK. A survey of one CCFP-EM program's graduates: their background, intended type of practice and actual practice. CFEM 2005;7: 315-20.

11. Eisenhardt KM, Graebner ME. Theory building from cases: opportunities and challenges. Acad Manage 7 2007;50. doi: 10.5465/amj.2007.24160888.

12. Yin RK. Applications of case study research. 3rd ed. Thousand Oaks (CA): SAGE Publications; 2011.

13. Vision, mission, values and goals. Willowdale (ON): College of Family Physicians of Canada; 1989. Available: https://www.cfpc.ca/principles (accessed 2021 May 18).

14. Hoffman A, Montgomery R, Aubry W, et al. How best to engage patients, doctors, and other stakeholders in designing comparative effectiveness studies. Health Aff (Millwood) 2010;29:1834-41.

15. Grierson L, Vanstone $M$. The rich potential for education research in family medicine and general practice. Adv Health Sci Educ Theory Pract 2021;26:753-63.

Competing interests: Catherine Tong reports a stipend for work done on the planning committee of the Ontario College of Family Physicians annual conference. Margo Mountjoy reports financial support and honoraria from the International Olympic Committee, the Fédération Internationale de Natation, the World Anti-Doping Agency, World Rugby and numerous other international sporting agencies for travel to support medical programs at international competitions. She has fulfilled unpaid leadership roles at the Fédération Internationale de Natation, the International Olympic Committee, World Rugby, the Fédération Internationale de Football Association and the Association of Summer Olympic International Federations. No other competing interests were declared.

Affiliations: Department of Family Medicine (Grierson, Allice, Baker, Farag, Guscott, Howard, Mountjoy, Siu, Tong, Vanstone) and McMaster Education Research, Innovation and Theory Program (Grierson, Vanstone), Faculty of Health Sciences, McMaster University, Hamilton, Ont.

Contributors: Lawrence Grierson and Meredith Vanstone supervised all aspects of the project, and conceived and designed the study. Ilana Allice obtained the data. Lawrence Grierson, Ilana Allice and Meredith Vanstone analyzed and interpreted the data, with contributions from Alison Baker, Alexandra Farag, Jesse Guscott, Michelle Howard, Margo Mountjoy, Henry Siu and Catherine Tong. Lawrence Grierson drafted the manuscript. All of the authors revised the manuscript critically for important intellectual content, approved the final version to be published and agreed to be accountable for all aspects of the work.

Funding: This work was supported by the College of Family Physicians of Canada.

Content licence: This is an Open Access article distributed in accordance with the terms of the Creative Commons Attribution (CC BY-NCND 4.0) licence, which permits use, distribution and reproduction in any medium, provided that the original publication is properly cited, the use is noncommercial (i.e., research or educational use), and no modifications or adaptations are made. See: https://creativecommons.org/licenses/by-nc $-\mathrm{nd} / 4.0 \%$.

Data sharing: These data are not available for use by other researchers.

Acknowledgements: The authors acknowledge the valuable contributions of the College of Family Physicians of Canada, and specifically Dr. Roy Wyman, Dr. Nancy Fowler, Dr. Jose Pereira, Tatjana Lozanovska and Danijela Stojanovska, for their support of this project.

Supplemental information: For reviewer comments and the original submission of this manuscript, please see www.cmajopen.ca/content/9/4/ E966/suppl/DC1. 